\title{
The Effect of Mitochondria in Intracellular Calcium Dynamics in Cardiomyocytes: a Simulation Study
}

\author{
Ainhoa Asensio, Jose M Ferrero \\ Universitat Politècnica de València, Valencia, Spain
}

\begin{abstract}
Intracellular calcium $\left(\mathrm{Ca}_{i}\right)$ regulation in cardiomyocytes is critical because of its relationship with cell contractility and arrhythmogenesis. Although the mitochondria play an important role in $\mathrm{Ca}_{i}$ regulation, few action potential (AP) models include these organelles. In this work, we have included mitochondria in an AP model of a rabbit cardiomyocyte to study its influence in $\mathrm{Ca}_{i}$ regulation. The model includes the calcium uniporter, the sodium-calcium exchanger and intra-mitochondrial calcium buffering. We conducted simulations in which $\mathrm{Ca}_{i}$ biomarkers were compared in the presence and in the absence of mitochondria. In some simulations, ionic currents were altered to simulate pharmacological interventions and/or the effect of mutations. Our results suggest that the absence of mitochondria in AP models introduces significant errors in the simulation of $\mathrm{Ca}_{i}$ handling, especially under conditions of altered currents.
\end{abstract}

\section{Introduction}

Intracellular calcium handling is a key process in the electrophysiology of cardiac myocytes $[1,2]$. On the one hand, it determines cell contractility [1], because intracellular calcium is the main regulator of myocardial excitation-contraction coupling. On the other hand, it is intimately related to arrhythmogenesis, because calcium overload may trigger abnormal cell depolarization [2,3]. The dynamic changes in intracellular calcium concentration $\left(\left[\mathrm{Ca}^{2+}\right]_{i}\right)$ during the action potential are complex. Upon cell depolarization at the onset of an action potential (AP), calcium enters the cell through the L-type calcium channels. This triggers a massive release of calcium from the sarcoplasmic reticulum (SR) to the cytosol through the ryanodine receptors, a process that may elevate $\left[\mathrm{Ca}^{2+}\right]_{\mathrm{i}}$ almost an order of magnitude compared to its basal value (the diastolic calcium level). After $\left[\mathrm{Ca}^{2+}\right]_{i}$ peaks (reaching its systolic level), it slowly declines due to (a) extrusion to the extracellular medium via the sodiumcalcium exchanger (NCX) in the sarcolemma, and (b) uptake to the SR via the SERCA pump [4].
Mitochondria are also known to play a role in intracellular calcium handling [5,6]. Indeed, mitochondria behave as calcium stores that can exchange this ion with the cytosol, at least via two calcium transport mechanisms present in the mitochondrion membrane: a calcium uniporter [7] and a mitochondrial sodium-calcium exchanger [8].

In the past two decades, mathematical modelling and computational simulations have been a powerful tool used to better understand the intricate mechanisms related to cellular electrophysiology (see [9] for review). These computational studies rely on detailed and comprehensive mathematical models of action potentials for different animal species [10-12], including human [13]. However, very few of these models include a formulation of the mitochondrial compartment and its effect on ion calcium dynamics. There is little theoretical knowledge of the error introduced by the absence of mitochondria in the models.

In this study, we have introduced a model of the mitochondrial calcium currents in a well-known existing model of the action potential and ionic currents of a rabbit ventricular myocytes [12]. We have used this integrated model to perform a sensitivity analysis to elucidate the ionic mechanisms that affect intracellular calcium regulation, both in the absence and in the presence of mitochondria. The results reveal that the absence of mitochondria in "classic" AP models introduces significant errors in the simulations.

\section{Methods}

The action potential, underlying ionic currents and dynamic changes in ionic concentrations of an isolated rabbit ventricular myocyte were simulated using a modified version of the Shannon-Bers model [12], which includes a very detailed formulation of calcium dynamics. On the one hand, a dynamic formulation of intracellular potassium concentration (which is fixed in the original model) was introduced. On the other hand, equations describing the influence of mitochondria on intracellular calcium handling were introduced in the model. Specifically, the model by Kim et al. [14] was used to formulate a computational model of the mitochondrial 
calcium uniporter ( $\mathrm{I}_{\text {uni,mit }}$ ), the mitochondrial sodiumcalcium exchanger $\left(\mathrm{I}_{\mathrm{NCX}, \mathrm{mit}}\right)$ and the intra-mitochondrial calcium buffering process. As the Kim et al. model is formulated for B lymphocytes, the parameters were adjusted for rabbit cardiomyocytes using data from $\mathrm{Lu}$ et al. [15]. Two different models (one without mitochondria (noMit model) and one with mitochondria (Mit model)) were used in the simulations.

A sensitivity analysis was performed by applying a oneat-a-time variation in the ionic currents through the sarcolemma and the SR membrane. Specifically, each current was multiplied by $0.05,0.5,1.0$ (no change) and 2.0 in order to study their impact on selected calciumrelated biomarkers. These changes could represent the effect of drugs or mutations, or simply inter-individual physiological variability [16]. All simulations were performed at $2 \mathrm{~Hz}$ and run until achieving steady-state. The measured calcium biomarkers included systolic $(\mathrm{CaS})$ and diastolic $(\mathrm{CaD})\left[\mathrm{Ca}^{2+}\right]_{\mathrm{i}}, \mathrm{Ca}^{2+}$ transient duration at $80 \%$ recovery (CaTD80), and calcium time-to-peak (CaTTP).

The sensitivity between the most severe current variation and the "no change" condition was calculated as explained in [17]. Finally, sensitivities were normalized to the maximum absolute sensitivity for each biomarker.

\section{Results and Discussion}

Prior to applying any change to ionic currents, we conducted a control simulation using both models (with and without mitochondria). The results, depicted in Fig. 1, show that the introduction of mitochondria has no noticeable effect in the AP waveform (panel A) but induces a slight change in the $\left[\mathrm{Ca}^{2+}\right]_{i}$ transient (panel $\mathrm{B}$ ). The impact in $\mathrm{CaS}$ is almost negligible $(0.3 \%)$, but $\mathrm{CaD}$ increases by $4.2 \%$ in the model with mitochondria. As for the time-related parameters, CaTD80 and CaTTP decrease by $2.8 \%$ and $5.8 \%$, respectively, in the model with mitochondria.

In order to investigate if these changes are enhanced when ionic currents are varied, the aforementioned sensitivity analysis was performed. A representative example of the results is shown in Fig. 2, where the $\left[\mathrm{Ca}^{2+}\right]_{i}$ transient is shown in control conditions and under almost total sarcolemmal NCX block (x0.05). The figure shows that the $\left[\mathrm{Ca}^{2+}\right]_{\mathrm{i}}$ transient is shifted upwards by blocking the NCX. The introduction of mitochondria in the model limits this shift, reducing the $\mathrm{CaS}$ by $9 \%$. As for the other biomarkers, $\mathrm{CaD}$ increases by $13 \%$, CaTTP decreases by $20 \%$ and CaTD 80 decreases by $4 \%$ when the mitochondria is present in the model. These percentages may be understood as errors introduced by the absence of mitochondria in the model.

The results obtained in the sensitivity analysis were then compiled and are represented in Fig. 3, which shows the values of the biomarker in the $\mathrm{Y}$ axis versus the current multiplying factor in the $\mathrm{X}$ axis for the four measured

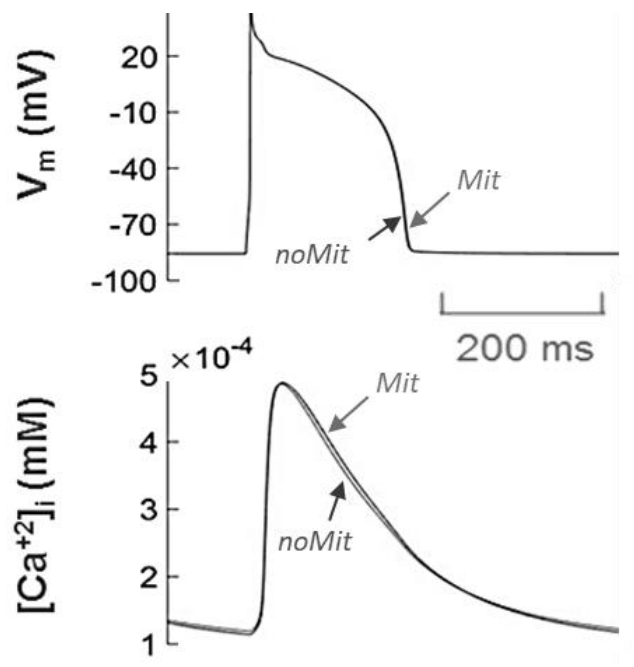

Figure 1. Action potentials (upper panel) and intracellular calcium transients (lower panel) in the presence and in the absence of mitochondria (Mit and noMit, respectively).

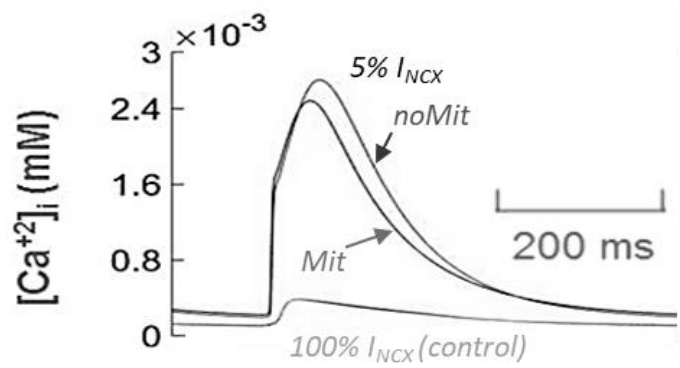

Figure 2. Intracellular calcium transients in the presence and in the absence of mitochondria (Mit and noMit, respectively). The two upper traces correspond to a $95 \%$ blockade of the sarcolemmal NCX current $\left(0.05 \mathrm{xI}_{\mathrm{NCX}}\right.$ in the model), while the two lower traces correspond to control $\left(100 \% \mathrm{I}_{\mathrm{NCX}}\right)$ simulations.

biomarkers. For the sake of clarity, only the effect of the three main currents that affect each biomarker are represented. Solid lines and dotted lines correspond to the results with and without the presence of mitochondria in the model, respectively.

Summarizing, the current whose variations most affect the calcium biomarkers is the SERCA current, except for $\mathrm{CaS}$ where the sarcolemmal NCX current exerts the stronger effect. Other currents that influence the calcium biomarkers when varying are the L-type calcium current and the SR release current through the RyR channels. In some cases, a noticeable difference is found between the noMit model and the Mit model, which is particularly significant for the NCX current and, in some cases, for the SERCA current as well.

Finally, the results mentioned above were normalized and expressed as relative sensitivities, as explained in [17]. For each biomarker, the current whose variations most 

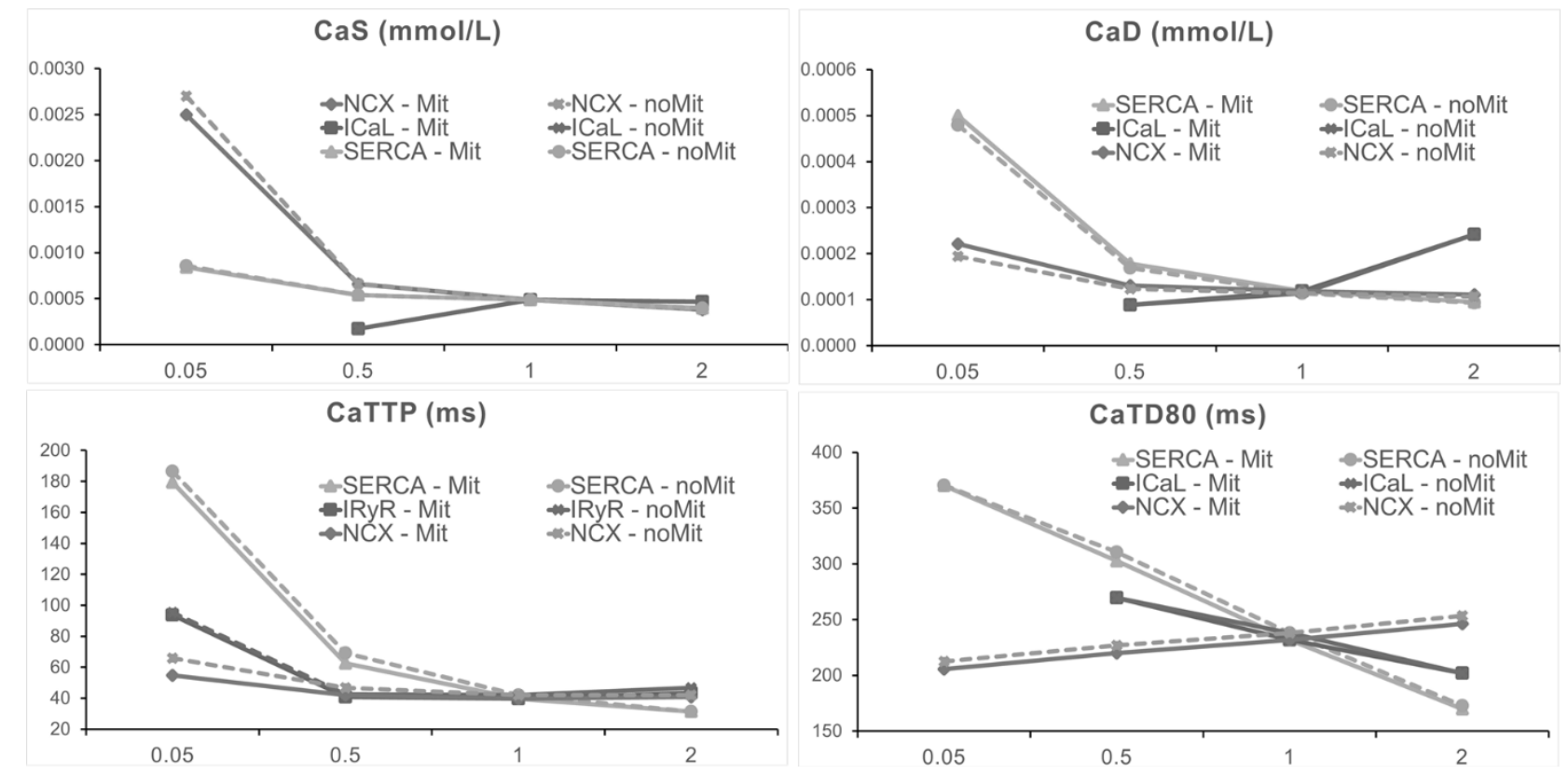

Figure 3. Compiled results of the sensitivity analysis. Each panel shows the variation of a biomarker as a function of the ionic current multiplying factor. Only the currents whose variations affect most are shown. Dotted lines correspond to the model without mitochondria, solid lines to the model including the organelle.

Table 1. Relative sensitivities of the calcium biomarkers to changes in ionic currents in the model without mitochondria.

\begin{tabular}{|lrrrrrrrrrr|}
\hline NoMit model & INCX & INa & INaK & ICaL & ICap & IK1 & IKs & IKr & SERCA & RyR \\
CaTTP & 15.4 & $\mathbf{4 . 2}$ & 1.6 & $\mathbf{2 . 1}$ & $\mathbf{0 . 5}$ & 1.1 & $\mathbf{0 . 8}$ & $\mathbf{0 . 2}$ & 100.0 & 31.4 \\
CaS & 100.0 & 0.5 & 18.1 & $\mathbf{1 6 . 5}$ & 1.4 & 0.8 & 2.0 & 15.3 & 19.7 & 3.5 \\
CaD & 22.6 & $\mathbf{0 . 2}$ & 7.6 & $\mathbf{5 1 . 5}$ & 0.6 & 0.7 & 0.9 & 6.4 & 100.0 & 23.6 \\
CaTD80 & $\mathbf{2 0 . 7}$ & $\mathbf{1 . 1}$ & 9.3 & $\mathbf{4 4 . 4}$ & 1.2 & $\mathbf{0 . 2}$ & 1.2 & 6.6 & 100.0 & $\mathbf{1 6 . 3}$ \\
\hline
\end{tabular}

Table 2. Relative sensitivities of the calcium biomarkers to changes in ionic currents in the model with mitochondria.

\begin{tabular}{|lrrrrrrrrrr|}
\hline Mit model & INCX & INa & INaK & ICaL & ICap & IK1 & IKs & IKr & SERCA & RyR \\
CaTTP & 9.3 & $\mathbf{4 . 7}$ & $\mathbf{0 . 6}$ & $\mathbf{2 . 2}$ & $\mathbf{0 . 2}$ & 1.3 & $\mathbf{0 . 9}$ & $\mathbf{2 . 3}$ & 100.0 & 34.0 \\
CaS & 100.0 & 0.5 & 19.4 & $\mathbf{1 8 . 0}$ & 1.5 & 0.9 & 2.2 & 16.5 & 21.1 & 3.6 \\
CaD & 27.3 & $\mathbf{0 . 2}$ & 8.3 & $\mathbf{4 9 . 1}$ & 0.7 & 0.7 & 1.0 & 7.1 & 100.0 & 22.4 \\
CaTD80 & $\mathbf{2 0 . 4}$ & $\mathbf{1 . 1}$ & 8.4 & 43.3 & 0.8 & 1.3 & 0.8 & 6.2 & 100.0 & $\mathbf{1 5 . 4}$ \\
\hline
\end{tabular}

affect the value of the biomarker is the $100 \%$ reference, so the effect of the variations of the other currents receive a value between 0 and $100 \%$. The results are compiled in Table 1 (for the model without mitochondria) and Table 2 (for the model with mitochondria). Bold numbers (in percentage) indicate a direct relationship (i.e. the biomarker increases with the multiplying factor), while italic numbers indicate and inverse relationship. Dark gray color indicates the strongest sensitivity per row, while white corresponds to a lack of dependency between biomarker and multiplying factor.

As can be seen in the tables (and also in Fig. 3), the
SERCA pump is the current to which three of the biomarkers (CaD, CaTTP and CaTD80) are most sensitive, while the NCX current plays that role in the case of CaS. NCX variations have a significant impact in $\mathrm{CaD}$ and CaTD80, while its effect in CaTTP is limited $(<10 \%)$ in the model with mitochondria. Variations in the L-type calcium current are also relevant for three of the biomarkers, with CaTTP again being an exception. The influence of the SR release current through the ryanodine receptors (RyR) is also significant in the biomarkers except for CaS. Variations in other membrane currents have a negligible influence on the biomarkers, except for the 
sodium-potassium pump in $\mathrm{CaS}$ and the rapid potassium current $\left(\mathrm{I}_{\mathrm{Kr}}\right)$ also in the systolic calcium level.

Inspection of the numbers in the tables reveals differences (i.e. errors) between the Mit and noMit models. The highest errors are found for the NCX current $(6.1 \%$ for CaTTP and $-4.7 \%$ for $\mathrm{CaD}$ ).

\section{Conclusion}

According to our results, the absence of mitochondria in an action potential model may introduce significant errors in the intracellular calcium transient characteristics. These errors are enhanced when altering the ionic currents and are higher under conditions of sarcolemmal NCX variations.

\section{Acknowledgements}

This work was partially supported by Dirección General de Política Científica de la Generalitat Valenciana (PROMETEO 2016/088), and by the "Plan Estatal de Investigación Científica y Técnica y de Innovación 20132016" from the Ministerio de Economía, Industria y Competitividad of Spain (DPI2016-75799-R) and AEI/FEDER, UE.

\section{References}

[1] Eisner DA, Caldwell JL, Kistamás K, Trafford AW. Calcium and Excitation-Contraction Coupling in the Heart. Circ Res. 2017 Jul 7;121(2):181-195.

[2] Ter Keurs HEDJ, Boyden PA. Calcium and Arrhythmogenesis. Physiol Rev. 2007 Apr; 87(2): 457506.

[3] Piacentino V, Weber CR, Chen X, Weisser-Thomas J, Margulies KB, Bers DM, et al. Cellular basis of abnormal calcium transients of failing human ventricular myocytes. Circ Res. 2003;92(6):651-8.

[4] Bers DM, Shannon TR. Calcium Movements Inside the Sarcoplasmic Reticulum of Cardiac Myocytes. J Mol Cell Cardiol. 2013 May; 58: 59-66.

[5] Murgia M, Giorgi C, Pinton P, Rizzuto R. Controlling metabolism and cell death: at the heart of mitochondrial calcium signalling. J Mol Cell Cardiol. 2009 Jun;46(6):781-8.

[6] Bers DM1, Bassani JW, Bassani RA. Competition and redistribution among calcium transport systems in rabbit cardiac myocytes. Cardiovasc Res. 1993 Oct;27(10):17727.

[7] Kirichok Y, Krapivinsky G, Clapham DE. The mitochondrial calcium uniporter is a highly selective ion channel. Nature. 2004 Jan 22;427(6972):360-4.
[8] Palty R, Silverman WF, Hershfinkel M, Caporale T, Sensi SL, Parnis J, Nolte C, Fishman D, Shoshan-Barmatz V, Herrmann S, Khananshvili D, Sekler I. NCLX is an essential component of mitochondrial $\mathrm{Na}^{+} / \mathrm{Ca}^{2+}$ exchange. Proc Natl Acad Sci U S A. 2010 Jan 5;107(1):436-41.

[9] Trénor B, Romero L, Cardona K, Gomis J, Saiz J, Ferrero JM. Multiscale Modeling of Myocardial Electrical Activity: From Cell to Organ. Applied Biomedical Engineering, Dr. Gaetano Gargiulo (Ed.), InTech, DOI: $10.5772 / 22907$.

[10] Luo CH, Rudy Y. A model of the ventricular cardiac action potential. Depolarization, repolarization, and their interaction. Circ Res. 1991 Jun;68(6):1501-26.

[11] Decker KF, Heijman J, Silva JR, Hund TJ, Rudy Y. Properties and ionic mechanisms of action potential adaptation, restitution, and accommodation in canine epicardium. 1D simulation study. Am J Physiol Heart Circ Physiol. 2009 Apr;296(4):H1017-26.

[12] Shannon TR, Wang F, Puglisi J, Weber C, Bers DM. A mathematical treatment of integrated $\mathrm{Ca}$ dynamics within the ventricular myocyte. Biophys J. 2004 Nov;87(5):335171.

[13] O’Hara T, Virág L, Varró A, Rudy Y. Simulation of the undiseased human cardiac ventricular action potential: Model formulation and experimental validation. PLoS Comput Biol. 2011;7(5).

[14] Kim B, Takeuchi A, Koga O, Hikida M, Matsuoka S. Pivotal role of mitochondrial $\mathrm{Na}^{+} \mathrm{Ca}^{2+}$ exchange in antigen receptor mediated $\mathrm{Ca}^{2+}$ signalling in DT40 and A20 B lymphocytes. J Physiol. 2012 Feb 1;590(3):459-74.

[15] Lu X1, Ginsburg KS, Kettlewell S, Bossuyt J, Smith GL, Bers DM. Measuring local gradients of intramitochondrial $[\mathrm{Ca}(2+)]$ in cardiac myocytes during sarcoplasmic reticulum $\mathrm{Ca}(2+)$ release. Circ Res. 2013 Feb $1 ; 112(3): 424-31$.

[16] Muszkiewicz A, Britton OJ, Gemmell P, Passini E, Sánchez C, Zhou X, Carusi A, Quinn TA, Burrage K, Bueno-Orovio A, Rodriguez B. Variability in cardiac electrophysiology: Using experimentally-calibrated populations of models to move beyond the single virtual physiological human paradigm. Prog Biophys Mol Biol. 2016 Jan;120(1-3):115-27.

[17] Romero L, Carbonell B, Trenor B, Rodríguez B, Saiz J, Ferrero JM. Systematic characterization of the ionic basis of rabbit cellular electrophysiology using two ventricular models. Prog Biophys Mol Biol. 2011 Oct;107(1):60-73.

Address for correspondence.

Chema Ferrero

Centro de Investigación e Innovación en Bioingeniería (Ci2B)

Universitat Politècnica de València

Camino de Vera s/n Valencia 46022, Spain

cferrero@ci2b.upv.es 